\title{
Cryptococcal infections of the central nervous system: an analysis of predisposing factors, laboratory findings and outcome in patients from South India with special reference to HIV infection
}

\author{
NEELAM KHANNA, A. CHANDRAMUKI, ANITA DESAI* and V. RAVI* \\ Departments of Microbiology and *Neurovirology, National Institute of Mental Health and Neuro Sciences, \\ Bangalore 560029, India
}

\begin{abstract}
The incidence of cryptococcosis in patients with AIDS is significant. Predisposing factors, laboratory findings and outcome were assessed in 60 patients with cryptococcal infections of the central nervous system over a 17.5-year period (Jan. 1978-June 1995). Predisposing factors for cryptococcal infection were identified in 36 patients, with HIV infection being the commonest (18). Cryptococcal cultures were positive in all patients. India ink staining was positive in 48 patients and cryptococcal antigen was detected in 35 of 36 patients tested. Comparison of clinical and laboratory parameters between HIV-positive and HIVnegative patients showed that CSF cell response was poorer, culture of cryptococci from non-neural sites was more frequent and mortality was higher in the HIV-positive group. Although not statistically significant, concurrent systemic infections, especially tuberculosis, were more frequent in the HIV-positive group.
\end{abstract}

\section{Introduction}

Cryptococcus neoformans is the commonest fungal pathogen to infect the human central nervous system (CNS) [1]. Cryptococcosis is increasing because of an ever rising population of immunocompromised patients, especially those with acquired immune deficiency syndrome (AIDS). It has been observed that in patients with AIDS, the clinical course and outcome of CNS cryptococcosis is unique $[1,2]$. Also, the incidence of cryptococcosis in AIDS patients in developing countries is much higher than that reported in developed countries [3].

\section{Materials and methods}

This study was conducted at the National Institute of Mental Health and Neurosciences Hospital which is the largest neuropsychiatric centre in India. It was retrospective and included all patients diagnosed as having cryptococcal meningitis over a 17.5-year period (Jan. 1978-June 1995). During this period, 85 culture-proven mycoses of the CNS were encountered at this centre.

Received 30 Nov. 1995; revised version accepted 4 March 1996.

Corresponding author: Professor A. Chandramuki.
Cryptococcal infections predominated, accounting for $70.5 \%(n=60)$ and there were 10 cases of aspergillosis, nine of zygomycosis and six of chromoblastomycosis. The diagnosis of cryptococcal meningitis was based on clinical features of chronic meningitis and laboratory findings such as demonstration of cryptococci by India ink staining and isolation of $C$. neoformans from the CSF or brain tissue. Clinical data and predisposing factors were recorded.

\section{Laboratory procedures}

All the CSF samples were analysed macroscopically for colour, turbidity and 'cobweb' formation and microscopically for the cell count with Neubauer's counting chamber. An India ink preparation of all the CSF samples was made to visualise the capsulate forms of cryptococci. Cytospin preparations were made whenever sufficient CSF was available, and the deposit was stained with Leishman's stain to look for budding yeast forms, pattern of inflammatory cell response and the presence of any malignant cells. Fungal cultures of all samples were performed with Sabouraud's dextrose agar (SDA). The cultures were incubated at $37^{\circ} \mathrm{C}$ and $25-27^{\circ} \mathrm{C}$. The latex agglutination test (LAT) (LatexCrypto Antigen Detection System, Immuno-Mycologics, Oklahama, USA) for detection of cryptococcal capsular polysaccharide antigen in the CSF was 
performed in 36 cases. Testing for associated HIV infection could be done in patients admitted after October 1989 (41 cases) with ELISA kits supplied under the National AIDS surveillance programme. Confirmation of HIV infection was done by either Western blot or triple ELISA according to WHO guidelines recommended for the developing nations [4].

\section{Results}

The medical records of 60 cases of cryptococcosis occurring between Jan. 1978 and June 1995 were analysed. The age of these patients ranged from 15 to 73 years (mean 35.9 years); 44 were male and 16 female. They originated from both urban and rural areas of the country and no occupational risk factors were identified. Twenty-four patients had no clinical evidence of underlying immunosuppression, whereas in 36 patients predisposing factors or associated illness, or both, suggested an immunocompromised state (Table 1). Of the 41 patients tested, $18(43.9 \%)$ were found to be infected with HIV. Tuberculosis, either pulmonary or systemic, was noted in $38.3 \%$ ( 23 of 60 ) of the patients. An increase in the incidence of cryptococcal meningitis was noted from 1991 onwards (Fig. 1) and this paralleled the increase in the number of AIDS cases. It is noteworthy that cryptococcal meningitis was the AIDS defining illness in these cases, although other opportunist infections were also present.

\section{Laboratory findings}

CSF analysis was performed in 58 cases for a definitive diagnosis. In two cases where the clinical presentation was suggestive of a space-occupying lesion, the diagnosis was based on culture and histopathological
Table 1. Predisposing factors identified on clinical and laboratory examination in 60 cases of cryptococcal meningitis

\begin{tabular}{lc}
\hline Factor & Number of patients \\
\hline HIV infection & 18 \\
Tuberculosis & \\
$\quad$ pulmonary & 10 \\
$\quad$ lymphadenitis & 1 \\
Pregnancy & 2 \\
Metastatic disease & 1 \\
Renal transplantation & 1 \\
Corticosteroids & 1 \\
Chronic alcoholism & 1 \\
Hypertension and diabetes & 1 \\
No known predisposing factor & 24 \\
Total & 60 \\
\hline
\end{tabular}

* Of the 18 patients with HIV infection, eight had associated pulmonary tuberculosis, one each had tubercular pleural effusion, lymphadenitis, meningitis and Bartholin's abscess and there was one case of liver haemangioma; five did not have any associated illness.

examination of resected tissue. CSF was observed to be clear in $63 \%$ of the cases, but hazy in $35 \%$. CSF leucocyte counts were within normal limits in $23.6 \%$ of patients and elevated in $76.4 \%$. The CSF cell counts ranged from 0 to 1200 cells $/ \mathrm{mm}^{3}$, with a mean cell count of 227.7 cells $/ \mathrm{mm}^{3}$. The predominant cell type was lymphocyte ( 30 of 55 cases) but both lymphocytes and neutrophils were observed in 23 and neutrophils only in two cases. Capsulate yeast forms were also seen in the counting chamber in some cases. In twelve patients the India ink preparations were negative for cryptococci on initial CSF examination but other methods such as culture, cytospin and cryptococcal LAT gave positive results. Fungal culture was positive in all cases within $72 \mathrm{~h}$ of inoculation, except for three cases where growth was observed only after 7 days. Of these three cases, the India ink preparation was positive in two and the third had a positive cryptococcal antigen test. All the isolates were identified as C. neoformans

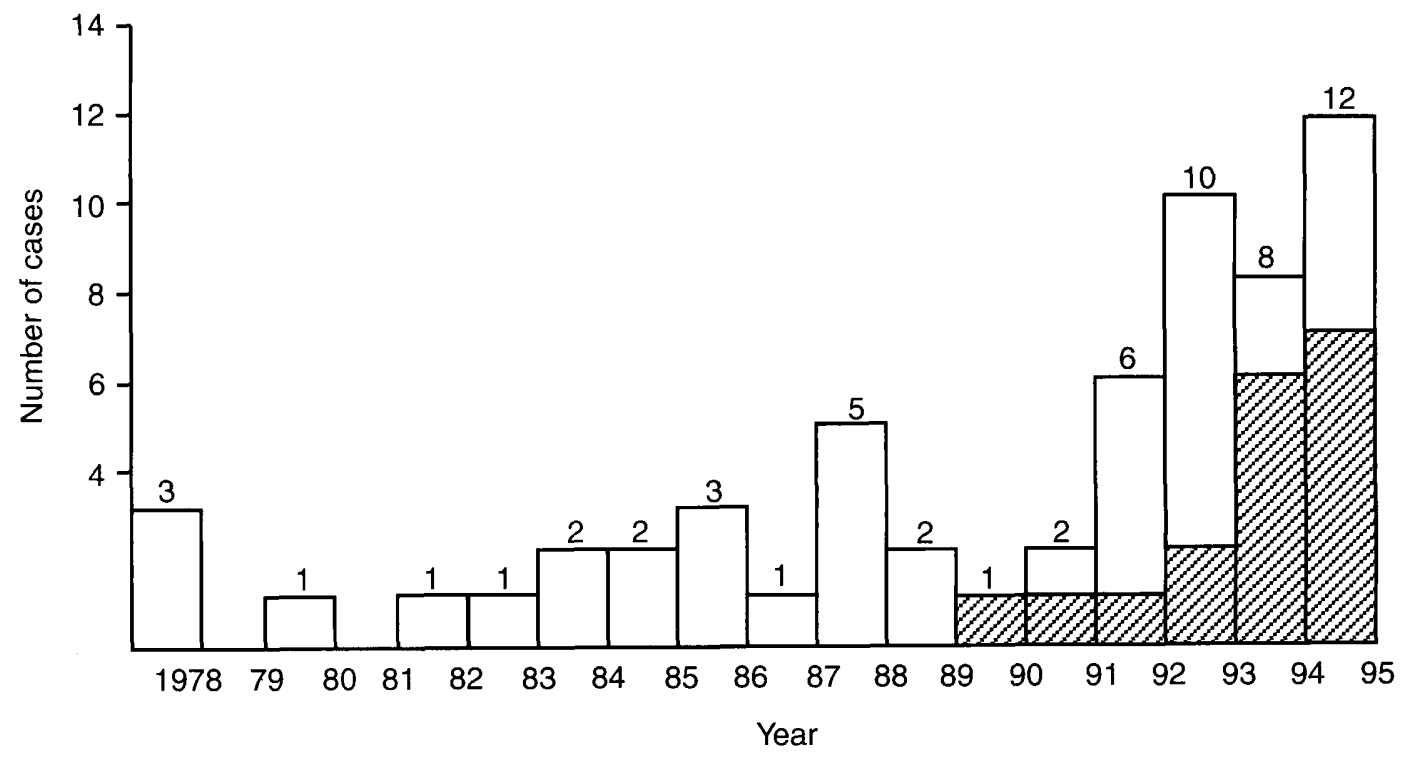

Fig. 1. Incidence of cryptococcal infections of the CNS. Note the increase in the number of cryptococcal infections seen from 1991 paralleling the increase in AIDS cases (). 
based on their moist, creamy appearance on SDA (both at $25^{\circ}$ and $37^{\circ} \mathrm{C}$ ), the capacity to produce phenol oxidase on caffeic acid agar, and urease production on Christensen's urea medium. In 10 patients, cryptococci were also isolated from other body fluids (urine, six; blood, five; gastric lavage, four and sputum, five).

The LAT for cryptococcal capsular polysaccharide antigen was performed in 36 cases and gave positive results in 35 . In one case the antigen could not be detected, although India ink preparation and fungal culture were positive. The presence of immune complexes (according to the manufacturer's procedure) and prozone phenomenon were ruled out in this case, which may suggest that it was a very early infection.

Analysis of repeat samples of CSF in patients revealed the persistence of pleocytosis with a lymphocytic predominance for up to 4 weeks. India ink preparations gave negative results in the initial culturepositive CSF samples of 12 patients but became positive in all these patients subsequently, either in the second $(n=8)$ or third sample $(n=4)$. In 26 patients from whom more than two CSF samples were available, cryptococci were found to persist microscopically in 22 cases, despite appropriate therapy.

Thirty-six patients were treated with amphotericin B and 5-flucytosine whilst 10 received fluconazole in addition to amphotericin B. The remaining 14 patients did not receive any antifungal therapy because they were discharged before a fungal aetiology was established. Amphotericin B was given as an intravenous infusion at a dose of $1 \mathrm{mg} / \mathrm{kg} /$ day. 5-Flucytosine was administered orally $(150 \mathrm{mg} / \mathrm{kg} /$ day $)$. In some patients, hyaluronidase (1500 units/week) was given intrathecally as an adjuvant [5] to anti-tubercular and antifungal therapy. The observation of four consecutive CSF samples negative for cryptococci by culture was taken as the criterion for stopping anti-fungal treatment.

\section{Outcome}

Of the 60 patients, 23 succumbed to the illness whilst on anti-fungal treatment or before specific treatment could be initiated, and an autopsy was performed on 12 of them. The final clinical outcome was not available in 14 patients as they were discharged before a fungal aetiology could be confirmed, or they left the hospital against medical advice. In 23 patients who survived the infection, 14 improved on treatment while nine showed little or no improvement in their clinical status.

To investigate the modulating role of HIV infection on the clinical course and outcome for patients with cryptococcal meningitis, the following parameters were compared between those with and without HIV infection: CSF cell response, direct microscopy by India ink method, cryptococcal antigen in the CSF, culture of cryptococci from extra-neural sites, presence of other associated systemic infections and final clinical outcome (fatal versus non-fatal). This analysis was restricted to 41 of 60 patients in whom HIV testing was performed. As evident from Table 2, there were significant differences between these two groups with respect to CSF cell response $(p=0.012)$, culture of cryptococci from extra-neural sites $(p=0.008)$ and a fatal outcome $(p=0.05)$

\section{Discussion}

Cryptococcosis has been described as an opportunist infection but is also known to affect apparently healthy individuals [6]. In the present series, 36 patients had predisposing or associated conditions amongst which HIV infection predominated (Table 1). HIV infection was first detected in India in 1985 and since then has spread rapidly, particularly in the last 5 years. According to a recent report from the National AIDS Control Organisation, India (July 1995), there are over 19432 detected HIV infections in this country, the seropositivity rate being $0.73 \%$. Of these, 1891 have developed AIDS.

Table 2. Comparison of clinical and laboratory parameters in 41 cryptococcal meningitis patients with and without HIV infection

\begin{tabular}{|c|c|c|c|c|}
\hline Parameter & & $\begin{array}{l}\text { HIV-positive group } \\
\qquad(\mathrm{n}=18)\end{array}$ & $\begin{array}{l}\text { HIV-negative group } \\
\qquad(\mathrm{n}=23)\end{array}$ & $\mathrm{p}$ value* \\
\hline Age (years) & $<50$ & 17 & 21 & 0.59 \\
\hline$(n=41)$ & $>50$ & 1 & 2 & NS \\
\hline CSF cells & $<20 / \mathrm{mm}^{3}$ & 9 & 3 & 0.01 \\
\hline$(n=41)$ & $>20 / \mathrm{mm}^{3}$ & 9 & 20 & \\
\hline India Ink staining & + & 13 & 18 & 0.46 \\
\hline$(n=41)$ & - & 5 & 5 & NS \\
\hline Cryptococcal antigen & + & 16 & 19 & 0.55 \\
\hline$(\mathrm{n}=36)$ & - & 0 & 1 & NS \\
\hline Culture from extra-neural & + & 6 & 1 & 0.01 \\
\hline sites $(n=27)$ & - & 5 & 15 & \\
\hline Associated systemic infections & Present & 13 & 11 & 0.10 \\
\hline$(n=41)$ & Absent & 5 & 12 & NS \\
\hline Outcome & Died & 9 & 4 & 0.05 \\
\hline$(\mathrm{n}=31)$ & Survived & 6 & 12 & \\
\hline
\end{tabular}

Figures in parentheses indicate the number of patients evaluated for each parameter. NS, not significant; + , positive; - , negative.

*A Fisher's exact probability test was used. 
The success of laboratory confirmation of CNS cryptococcal infection achieved in the present study is attributable to the use of three laboratory methods direct microscopy, culture and antigen detection. Every patient clinically diagnosed with chronic meningitis should be screened for fungal infections by rapid methods, although fungal culture remains the gold standard. Specimens should be incubated for 2 weeks before being reported as negative. Amongst the rapid methods, the LAT for polysaccharide antigen is highly sensitive and specific [7,8] and is useful both in detecting $C$. neoformans infection and in monitoring response to therapy. False negative CSF LAT has been reported [9] and occurred in settings of low cryptococcal antigen concentrations which are more likely to be associated with non-AIDS case [10]. This can result in delayed diagnosis and therapy. A negative LAT result does not exclude the diagnosis of cryptococcal infection [10] and it is strongly recommended that fungal cultures should also be performed.

Quantitative studies of antigen titres in patients on treatment were not done in this study for economic reasons. However, a decrease in the grade of agglutination was noted in the non-HIV cases responding to therapy, unlike the HIV group where no change was observed despite adequate therapy.

The presentation of HIV infected patients with $C$. neoformans meningitis is characterised by the lack of meningeal signs, diminished inflammatory response, positive India ink smears, positive LAT for cryptococcal antigen, and the isolation of cryptococci from extra-neural sites [2, 11-13]. The CSF indices (cell count, protein and glucose) are almost always abnormal in non-HIV patients with cryptococcal meningitis while they may be normal or only mildly abnormal in patients with AIDS. In all patients, organism-specific studies on CSF - India ink preparation and cryptococcal antigen - are usually positive, indicating an increased fungal load with diminished host response [1, 13]. This study confirmed (Table 2) that a poor CSF cellular response of $<20$ cells $/ \mathrm{mm}^{3}$ was more common in the HIV-positive group ( $p=0.012$ ); however, there was no difference in the India ink positivity between the two groups. The greater frequency with which $C$. neoformans was isolated from extra-neural sites in the HIV positive group was also significant, as reported by others [2, $11,13,14]$. Mortality was higher in the HIV infected group of patients as compared to the HIV negative group $(\mathrm{p}=0.05)$ despite prompt anti-fungal therapy.
Although not significantly different, it was observed that the occurrence of other associated systemic infections, especially tuberculosis, was more frequent in the HIV-positive group $(72 \%)$ than in the negative group $(48 \%)$. This finding has not been reported previously from developed nations $[2,12,13]$. Tuberculosis is the commonest secondary infection in AIDS patients in India [14] and, therefore, it is not surprising that it was seen more often in the HIVpositive group. One hundred years after the discovery of $C$. neoformans, mechanisms of virulence remain unresolved. Advances in methods of diagnosis, development of vaccines and improved anti-fungal therapy will probably help to control this epidemic of cryptococcosis.

The authors gratefully acknowledge the support of the clinicians and the Director, NIMHANS.

\section{References}

1. Dismukes WE. Cryptococcal meningitis in patients with AIDS. $J$ Infect Dis 1988; 157: 624-628.

2. Chuck SL, Sande MA. Infections with Cryptococcus neoformans in the acquired immunodeficiency syndrome. $N$ Engl $J$ Med 1989; 321: 794-799.

3. Kozel TR. Virulence factors of Cryptococcus neoformans. Trends Microbiol 1995; 3: 295-299.

4. Training module on the sentinel surveillance of HIV infection. National AIDS Control Organisation, Ministry of Health and Family Welfare, Govt of India, June 1993

5. Gourie-Devi M, Satishchandra P. Hyaluronidase as an adjuvant in the management of tuberculous spinal arachnoiditis. $J$ Neurol Sci 1991; 102: 105-111.

6. Yu YL, Lau YN, Woo E, Wong KL, Tse B. Cryptococcal infection of the nervous system. $Q J \mathrm{Med} 1988$; 66: 87-96.

7. Kaufman L, Blumer S. Value and interpretation of serological tests for the diagnosis of cryptococcosis. Appl Microbiol 1968; 16: $1907-1912$.

8. Goodman JS, Kaufman L, Koenig MG. Diagnosis of cryptococcal meningitis. Value of immunological detection of cryptococcal antigen. $N$ Engl $J$ Med 1971; 285: 434 436.

9. Currie BP, Freundlich LF, Soto MA, Casadevall A. Falsenegative cerebrospinal fluid cryptococcal latex agglutination tests for patients with culture-positive cryptococcal meningitis. $J$ Clin Microbiol 1993; 31: 2519-2522.

10. Perfect JR. Cryptococcosis. Infect Dis Clin North Am 1989; 3: 77-102.

11. Mitchell TG, Perfect JR. Cryptococcosis in the era of AIDS 100 years after the discovery of Cryptococcus neoformans. Clin Microbiol Rev 1995; 8: 515-548.

12. Kwon-Chung $\mathrm{KJ}$, Bennett JE. Epidemiologic differences between the two varieties of Cryptococcus neoformans. Am J Epidemiol 1984; 120: 123-130.

13. Clark RA, Greer D, Atkinson W, Valainis GT, Hyslop N. Spectrum of Cryptococcus neoformans infection in 68 patients infected with human immunodeficiency virus. Rev Infect Dis 1990; 12: 768-777.

14. Kaur A, Babu PG, Jacob $\mathrm{M}$ et al. Clinical and labóratory profile of AIDS in India. J Acquir Immune Defic Syndr 1992; 5: $883-889$. 